\title{
Perioperative Management of ACE Inhibitor Therapy: Challenges of Clinical Decision Making Based on Surrogate Endpoints
}

\author{
Duminda N. Wijeysundera MD, PhD, FRCPC $1,2,3 *$
}

'Li Ka Shing Knowledge Institute, St. Michael's Hospital, Toronto, Ontario, Canada; ${ }^{2}$ Department of Anesthesia and Pain Management, Toronto General Hospital, Toronto, Ontario, Canada; ${ }^{3}$ Department of Anesthesia, University of Toronto, Toronto, Ontario, Canada.

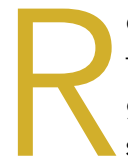

enin-angiotensin inhibitors, which include angiotensin-converting enzyme (ACE) inhibitors and angiotensin II receptor blockers (ARBs), have demonstrated benefits in the treatment of several common cardiovascular and renal conditions. For example, they are prescribed to individuals with hypertension, heart failure with reduced ejection fraction (HFrEF), prior myocardial infarction, and chronic kidney disease with proteinuria. Perhaps unsurprisingly, many individuals presenting for surgery are already on long-term ACE inhibitor or ARB therapy. For example, such individuals comprised approximately one-third of the sample in the Vascular Events In Noncardiac Surgery Patients Cohort Evaluation (VISION) multicenter prospective cohort study of major inpatient noncardiac surgery. ${ }^{1}$

There is considerable controversy regarding how best to manage these cardiovascular medications during the perioperative period. The critical question pertains to whether renin-angiotensin inhibitors should be temporarily withdrawn 24 hours before surgery or continued uninterrupted up to the day of surgery. The main argument for withdrawing these medications is concern that they cause perioperative hypotension. For example, a recent systematic review of randomized controlled trials (RCTs) and cohort studies found that preoperative continuation of renin-angiotensin inhibitor therapy led to a significantly increased risk of intraoperative hypotension, albeit without associated effects on rates of death, major adverse cardiac events, or postoperative hypotension. ${ }^{2}$ Notably, randomized trial evidence in this meta-analysis was limited to only five trials with a total of 774 participants. Conversely, preoperative interruption of renin-angiotensin inhibitor therapy also has risks. For example, there is a potential for unintended permanent discontinuation of medications with longterm benefits. ${ }^{3}$ Furthermore, some prior cohort studies have demonstrated that the failure to resume renin-angiotensin inhibitor therapy promptly after surgery is associated with an elevated risk of postoperative mortality. ${ }^{4,5}$ While these studies have methodological limitations related to survivorship bias and unmeasured confounders, they still raise concerns that the abrupt withdrawal of long-term cardiovascular therapy before

*Address for correspondence: Duminda N. Wijeysundera, MD PhD FRCPC; Li Ka Shing Knowledge Institute, St. Michael's Hospital, 30 Bond Street, Toronto, Ontario Canada M5B 1W8; E-mail: d.wijeysundera@utoronto.ca

Published online first July 25, 2018.

Received: June 3, 2018; Accepted: June 5, 2018

๑) 2018 Society of Hospital Medicine DOI 10.12788/jhm.3062 major surgery can have adverse effects. While ACE inhibitor withdrawal has not shown adverse physiological effects in the perioperative setting, it has led to rebound myocardial ischemia in patients with prior myocardial infarction. ${ }^{6}$

Given this controversy, there is variation across hospitals ${ }^{1}$ and practice guidelines with respect to perioperative management of renin-angiotensin inhibitors. For example, the 2017 Canadian Cardiovascular Society guidelines recommend that renin-angiotensin inhibitors be stopped temporarily 24 hours before major inpatient surgery, ${ }^{7}$ and the 2014 European guidelines recommend continuing therapy in patients with HFrEF but temporarily interrupting therapy in patients with hypertension. ${ }^{8}$ The 2014 American Heart Association and American College of Cardiology guidelines suggest that either continuation or interruption are reasonable options, but any interrupted therapy should be restarted postoperatively as soon as clinically feasible.?

In this issue of the Journal of Hospital Medicine, Shiffermiller and colleagues present a single-center RCT that provides additional high-quality data to improve our understanding of this important clinical issue..$^{10}$ In a sample of 275 patients undergoing nonvascular inpatient noncardiac surgery, omission of the final dose of preoperative ACE inhibitor therapy reduced the risk of intraoperative hypotension across multiple definitions, including any episode of systolic blood pressure less than 80 $\mathrm{mm} \mathrm{Hg}$ (number needed to treat: 8), any episode of a systolic blood pressure less than $80 \mathrm{~mm} \mathrm{Hg}$ necessitating vasopressor therapy (number needed to treat: 6), and total cumulative duration of intraoperative systolic blood pressure less than 80 $\mathrm{mm} \mathrm{Hg}$. In addition, the investigators found that preoperative interruption of ACE inhibitor therapy reduced the risk of postoperative hypotension (number needed to treat: 9), increased the risk of severe postoperative hypertension (number needed to harm: 9), and had no effect on clinical outcomes (eg, acute kidney injury, major adverse cardiac events). In conjunction with a recent systematic review, ${ }^{2}$ these new data demonstrate that temporary preoperative discontinuation of renin-angiotensin inhibitors leads to reduced risks of intraoperative and postoperative hypotension, with the only major identified risk being episodes of postoperative hypertension.

This current evidence base suggests that, in most cases, perioperative physicians should temporarily interrupt renin-angiotensin inhibitor therapy before inpatient noncardiac surgery, provided that protocols are in place to resume treatment postoperatively as soon as clinically feasible. Nonetheless, clinicians must also be cognizant of the key limitations to current data, 
namely that hypotension, be it intraoperative or postoperative, remains essentially a surrogate endpoint. ${ }^{11,12}$ Stated otherwise, the clinical importance of perioperative hypotension is largely predicated on its close association with clinically important or patient-relevant outcomes such as cardiovascular complications, acute kidney injury, and death. ${ }^{13-16}$ There is an implicit assumption that a reduction in the risk of hypotension will necessarily lead to reduced rates of clinical adverse events. This assumption is unlikely to be true, especially since many different underlying mechanisms lead to hypotension in the dynamic perioperative environment, including decreased cardiac contractility, decreased heart rate, decreased intravascular volume status, and vasodilation. Consistent with this possibility, different perioperative interventions with similar effects on hypotension have shown quite different effects on clinical outcomes. For example, epidural analgesia invariably reduces perioperative blood pressure, yet it does not appear to increase the risk of postoperative complications. ${ }^{17}$ Similarly, both beta-blockers and clonidine increase the risk of significant perioperative hypotension and bradycardia, yet only beta-blockers appear to lead to increased rates of mortality after noncardiac surgery. ${ }^{18,19}$ Thus, the relationship between perioperative hypotension and outcomes is clearly complex. Unless a RCT demonstrates that a hypotension-reduction strategy leads to an improvement in clinical outcomes, ${ }^{20}$ perioperative physicians should not assume that prevention of hypotension will always lead to improvements in patient-relevant clinical outcomes. Similar assumptions about other surrogate endpoints in cardiovascular medicine have sometimes been spectacularly incorrect. ${ }^{12,21}$ To more definitively address this important clinical issue, RCTs must be specifically designed to compare the effects of renin-angiotensin inhibitor therapy withdrawal versus continuation on patient-relevant and clinically important outcomes, such as death, myocardial infarction, and stroke. Fortunately, some ongoing trials will address this question, either directly (ClinicalTrials.gov NCT03374449) or as a component of a hypotension-avoidance strategy (ClinicalTrials.gov NCT03505723).

Overall, perioperative physicians should now adopt the standard approach of temporarily withdrawing renin-angiotensin inhibitor therapy 24 hours before major inpatient noncardiac surgery. Nonetheless, they should do so cautiously, recognizing that the data underpinning this strategy remain weak. As with many aspects of perioperative medicine, more research remains needed.

Disclosures: The authors have nothing to report.

Funding: DNW is supported in part by a New Investigator Award from the Canadian Institutes of Health Research, and a Merit Award from the Department of Anesthesia at the University of Toronto.

\section{References}

1. Roshanov PS, Rochwerg B, Patel A, et al. Withholding versus continuing angiotensin-converting enzyme inhibitors or angiotensin II receptor blockers before noncardiac surgery: An Analysis of the Vascular events In noncardiac Surgery patients cohort evaluation prospective cohort. Anesthesiology. 2017;126(1):16-27. doi: 10.1097/ALN.0000000000001404.

2. Hollmann C, Fernandes NL, Biccard BM. A systematic review of outcomes associated with withholding or continuing angiotensin-converting enzyme inhibitors and angiotensin receptor blockers before noncardiac surgery [published online ahead of print January 29, 2018]. Anesth Analg. doi: 10.1213/ANE.0000000000002837.

3. Bell CM, Bajcar J, Bierman AS, Li P, Mamdani MM, Urbach DR. Potentially unintended discontinuation of long-term medication use after elective surgical procedures. Arch Intern Med. 2006;166(22):2525-2531. doi: 10.1001/ archinte.166.22.2525.

4. Mudumbai SC, Takemoto S, Cason BA, Au S, Upadhyay A, Wallace AW. Thirty-day mortality risk associated with the postoperative nonresumption of angiotensin-converting enzyme inhibitors: a retrospective study of the Veterans Affairs Healthcare System. J Hosp Med. 2014;9(5):289-296. doi: 10.1002/jhm.2182.

5. Lee SM, Takemoto S, Wallace AW. Association between withholding angiotensin receptor blockers in the early postoperative period and 30-day mortality: a cohort study of the Veterans Affairs Healthcare System. Anesthesiology. 2015;123(2):288-306. doi: 10.1097/ALN.0000000000000739.

6. van den Heuvel AF, van Gilst WH, van Veldhuisen DJ, de Vries RJ, Dunselman $\mathrm{PH}$, Kingma JH. Long-term anti-ischemic effects of angiotensin-converting enzyme inhibition in patients after myocardial infarction. J Am Coll Cardiol. 1997;30(2):400-405. doi: 10.1016/S0735-1097(97)00183-6

7. Duceppe E, Parlow J, MacDonald P, et al. Canadian Cardiovascular Society guidelines on perioperative cardiac risk assessment and management for patients who undergo noncardiac surgery. Can J Cardiol. 2017;33(1):17-32. doi: 10.1016/j.cjca.2016.09.008

8. Kristensen SD, Knuuti J, Saraste A, et al. 2014 ESC/ESA Guidelines on non-cardiac surgery: cardiovascular assessment and management./ The Joint Task Force on non-cardiac surgery: cardiovascular assessment and management of the European Society of Cardiology (ESC) and the European Society of Anaesthesiology (ESA). Eur Heart J. 2014;35(35):2383-2431. doi: 10.1093/eurheartj/ehu282.

9. Fleisher LA, Fleischmann KE, Auerbach AD, et al. 2014 ACC/AHA guideline on perioperative cardiovascular evaluation and manage-

ment of patients undergoing noncardiac surgery: a report of the American College of Cardiology/American Heart Association Task Force on Practice Guidelines. Circulation. 2014;130(24):e278-e333. doi: 10.1161/ CIR.0000000000000105

10. Shiffermiller JF, Monson BJ, Vokoun CW, et al. Prospective randomized evaluation of preoperative angiotensin converting enzyme inhibition (PREOPACEI. J Hosp Med. 2018;13(10):661-667. doi: 10.12788/jhm.3036.

11. Psaty BM, Weiss NS, Furberg CD, et al. Surrogate end points, health outcomes, and the drug-approval process for the treatment of risk factors for cardiovascular disease. JAMA. 1999;282(8):786-790. doi: 10.1001/ jama.282.8.786.

12. Vanderweele TJ. Surrogate measures and consistent surrogates. Biometrics. 2013;69(3):561-569. doi: 10.1111/biom.12071.

13. Sun LY, Wijeysundera DN, Tait GA, Beattie WS. Association of intraoperative hypotension with acute kidney injury after elective noncardiac surgery. Anesthesiology. 2015;123(3):515-523. doi: 10.1097/ALN.0000000000000765.

14. van Waes JA, van Klei WA, Wijeysundera DN, van Wolfswinkel L, Lindsay TF, Beattie WS. Association between intraoperative hypotension and myocardial injury after vascular surgery. Anesthesiology. 2016;124(1):35-44. doi: 10.1097/ALN.0000000000000922.

15. Salmasi V, Maheshwari K, Yang D, et al. Relationship between intraoperative hypotension, defined by either reduction from baseline or absolute thresholds, and acute kidney and myocardial injury after noncardiac surgery: a retrospective cohort analysis. Anesthesiology. 2017;126(1):47-65. doi: 10.1097/ ALN.0000000000001432

16. Monk TG, Bronsert MR, Henderson WG, et al. Association between intraoperative hypotension and hypertension and 30-day postoperative mortality in noncardiac surgery. Anesthesiology. 2015;123(2):307-319.doi: 10.1097/ ALN.0000000000000756.

17. Rigg JR, Jamrozik K, Myles PS, et al. Epidural anaesthesia and analgesia and outcome of major surgery: a randomised trial. Lancet. 2002;359(9314):12761282. doi: 10.1016/S0140-6736(02)08266-1.

18. POISE Study Group. Effects of extended-release metoprolol succinate in patients undergoing non-cardiac surgery (POISE trial): a randomised controlled trial. Lancet. 2008;371(9627):1839-1847. doi: 10.1016/S0140-6736(08)60601-7.

19. Devereaux PJ, Sessler DI, Leslie K, et al. Clonidine in patients undergoing noncardiac surgery. N Engl J Med. 2014;370(16):1504-1513. doi: 10.1056/NEJMoa1401106 
20. Futier E, Lefrant JY, Guinot PG, et al. Effect of individualized vs standard blood pressure management strategies on postoperative organ dysfunction among high-risk patients undergoing major surgery: a randomized clinical trial. JAMA. 2017;318(14):1346-1357. doi: 10.1001/jama.2017.14172.
21. Cardiac Arrhythmia Suppression Trial (CAST) Investigators. Preliminary report: effect of encainide and flecainide on mortality in a randomized trial of arrhythmia suppression after myocardial infarction. N Engl J Med. 1989;321(6):406-412. doi:10.1056/NEJM198908103210629 